\title{
Physicochemical and Preclinical Evaluation of a Novel Buccal Measles Vaccine
}

\author{
Rikhav P. Gala, ${ }^{1}$ Carmen Popescu, ${ }^{2}$ Gregory T. Knipp, ${ }^{3,7}$ Robyn R. McCain, ${ }^{4}$ Ruhi V. Ubale, ${ }^{1}$ Richard Addo, ${ }^{5}$ \\ Tuhin Bhowmik, ${ }^{6}$ Christopher D. Kulczar, ${ }^{3}$ and Martin J. D'Souza ${ }^{1,7}$
}

Received 21 January 2016; accepted 3 June 2016; published online 29 June 2016

\begin{abstract}
The aim of this study is to develop an orally disintegrating film (ODF) containing a microparticulate measles vaccine formulation for buccal delivery. The measles vaccine microparticles were made with biocompatible and biodegradable bovine serum albumin (BSA) and processed by spray drying. These vaccine microparticles were incorporated in the ODF, consisting of Lycoat RS720®, Neosorb P60W® and Tween 80. The yield of the microparticles was approximately $85-95 \%, w / w$. The mean size of the vaccine microparticles was $3.65 \pm 1.89 \mu \mathrm{m}$ and had a slightly negative surface charge of 32.65 $\pm 2.4 \mathrm{mV}$. The vaccine particles were nontoxic to normal cells at high concentrations $(500 \mu \mathrm{g} /$ $2.5 \times 10^{5}$ cells) of vaccine particles. There was a significant induction of innate immune response by vaccine microparticles which was observed in vitro when compared to blank microparticles $(P<0.05)$. The vaccine microparticles also significantly increased the antigen presentation and co-stimulatory molecules expression on antigen presenting cells, which is a prerequisite for Th1 and Th2 immune responses. When the ODF vaccine formulation was dosed in juvenile pigs, significantly higher antibody titers were observed after week 2 , with a significant increase at week 4 and plateauing through week 6 comparative to naïve predose titers. The results suggest that the ODF measles vaccine formulation is a viable dosage form alternative to noninvasive immunization that may increase patient compliance and commercial distribution.
\end{abstract}

KEY WORDS: buccal delivery; measles; microparticle; ODF; pediatric; vaccine.

\section{INTRODUCTION}

Measle is a highly contagious infectious disease caused by the measles virus and was responsible for high worldwide morbidity, with conservative estimates in the millions of patients, and potential mortality prior to the introduction of the measles vaccine $(1,2)$. The attenuated measles vaccines were introduced in 1960s after the successful isolation and culture of the measles virus in tissue culture by John Enders

\footnotetext{
${ }^{1}$ Vaccine Nanotechnology Laboratory, Department of Pharmaceutical Sciences, Mercer University, 3001 Mercer University Drive, Atlanta, GA 30341, USA.

${ }^{2}$ Roquette America Inc, Geneva, IL, USA.

${ }^{3}$ Department of Industrial and Physical Pharmacy, College of Pharmacy, Purdue University, 575 Stadium Mall Dr, West Lafayette, IN 47906, USA.

${ }^{4}$ Purdue Translational Pharmacology of Bindley Biosciences, Purdue University, West Lafayette, IN, USA.

${ }^{5}$ Department of Pharmaceutical Sciences, Union University School of Pharmacy, Jackson, TN, USA.

${ }^{6}$ Takeda Vaccine Business Unit, Bozeman, MT, USA.

${ }^{7}$ To whom correspondence should be addressed. (e-mail: gknipp@purdue.edu; dsouza_mj@mercer.edu)
}

and then subsequent attenuation by Maurice Hilleman $(2,3)$. With the advent of these vaccines, tremendous progress has been achieved in reducing the number of deaths due to measles. Nonetheless, patient exposure has been steadily increasing, culminating in a record outbreak of measles in 2015 (4). This is because the measles virus is one of the most highly contagious, directly transmitted pathogens, where outbreaks can occur in populations in which less than $10 \%$ of people (infants and adults) are susceptible (5).

With the current vaccination regimen, two doses of the measles vaccine are currently prescribed: one for infants before the age of 12 months and a second booster vaccine administered in between 5 and 10 years of age $(3,6)$. In the case of the measles vaccine, both humoral and cell-mediated immune responses are important in fighting measles virus infection $(7,8)$. The humoral response is critical in controlling viral replication and conferring protection, while the cellmediated immune response is necessary in overcoming acute measles infection by eliminating infected cells (8). Thus, for controlling an epidemic outbreak in the developed and developing countries, a noninvasive, easy to transport and administer vaccine is needed which correspondingly can activate both arms of the immune system and provide 
maximum protection $(9,10)$. This therapy has largely reduced the incidence of measles epidemics in infants, although in recent years, there has been a pronounced increase in outbreaks that have caused global alarm. It should also be noted that debate over the role of thimerosal in the etiology of autism and the related spectrum of disorders may have led to a reduction in measles vaccinations and could be partly responsible for the significant recurrence of measles in this age range (11-14). Therefore, currently, a significant research emphasis has been placed on the development of novel vaccine delivery systems that improve patient compliance through the use of noninvasive dosage forms that eliminate thimerosal in order to prevent outbreaks within the unvaccinated population, thus raising herd immunity to a more effective level $(3,6)$.

An orally disintegrating film (ODF) formulation is a thin film prepared using hydrophilic polymers which dissolve rapidly on the tongue in the mouth (15). The buccal cavity provides a large surface area for rapid disintegration, release of the therapeutic entity, and subsequent absorption and provides a potentially good site for antigen delivery. The mucosal surface of the buccal cavity can be an ideal site for vaccination because of its easy accessibility and offers good antigen presentation $(16,17)$. The buccal cavity is rich in dendritic cells similar to Langerhans cells, which are a type of antigen-presenting cells (APC). Also, a high density of T lymphocytes and mucosal-associated lymphoid tissue like tonsils, salivary glands, Waldeyer's ring, and pharyngeal lymphoid tissue is present in the buccal mucosa (17). Hence, buccal immunization utilizing an ODF can help to elicit both mucosal and systemic immunity. ODF formulations are also very robust and enable the accurate delivery of selected antigens with ease of administration reflected in patient compliance for both pediatrics and adults (18-20). Moreover, ODF formulations may also provide safe delivery in infants since the rapid dissolution may alleviate dysphagia and associated choking concerns (21).

Mucosal vaccines have been more recently evaluated and have shown great success, suggesting that the approach can be further evaluated in the future (22). Previous studies have shown that microparticulate vaccine for delivery via the mucosal surfaces such as the oral cavity has elicited a significantly higher immune response compared to equivalent solution or suspension formulations (23-25). Thus, in this study, we have formulated the measles vaccine encapsulated in microparticles intended to be delivered via the buccal route. These microparticles are made from biodegradable material that slowly release antigens, thereby having an antigen depot effect to enhance immunogenicity (26-28). A potential advantage of utilizing the enteric polymer matrix is to protect the vaccine microparticles that have not been absorbed in the buccal cavity from the gastrointestinal environment. This will enable them to elicit an additional immune response in the lower small intestine and the ascending colon after traversing the GI Tract. A number of oral vaccines have also been developed to elicit a response in these regions. Antigen presentation to the immune cells such as the antigen presenting cells has been suggested to significantly improve response when given in a microparticulate form (29). Vaccine microparticles formulated as a dry powder possess extended shelf life and does not require cold-chain facilities for storage and commercial distribution and along with its formulation in a new dosage form such as the ODF (30) becomes compliant with a global distribution.

In order to overcome these challenges such as needle and syringe free delivery, and to enable ease of administration, we have developed a novel orally disintegrating film (ODF) formulation for microparticulate measles vaccine delivery that is readily adaptable to large-scale production. Moreover, the ODF will facilitate the first line of defense by providing mucosal exposure and triggering immunity through both systemic and local immune response toward the measles virus. In this study, we have formulated measles vaccine in a microparticulate form by using the spray drying technique. These microparticles were incorporated into an optimized orally disintegrating film to produce the ODF measles vaccine formulation for delivery via the buccal route. The ODF formulation of the embedded microparticles can also be readily taste masked to improve compliance, although we have not assessed those parameters in this proof of concept study. We have tested this novel ODF vaccine formulation in juvenile porcine model and found that significantly increased levels of antibody have been produced in contrast to naïve juvenile porcine controls. We have also demonstrated antigen presentation and induction of innate immunity in response to our vaccine in vivo. Thus, our data demonstrates that a novel measles vaccine delivered in a flexible, orally disintegrating film (ODF) formulation can induce an efficient immune response that may be translated for global clinical applications (Fig. 1).

\section{MATERIALS AND METHODS}

\section{Materials}

Live attenuated measles virus (Edmonston Zagreb Strain), M-VAC ${ }^{\text {TM }}$ in lyophilized form, was obtained from Serum Institute of India Ltd., Pune, India. Sterile and endotoxin-free bovine serum albumin (BSA) used to formulate the protein-based microparticles was purchased from Sigma-Aldrich. LYCOAT® RS 720, Neosorb P60W used to make the ODF, was kind gift from Roquette America Inc., Geneva, IL. Dendritic cells (DC2.4) were given as a kind gift from Dr. Kenneth L. Rock (Dana-Farber Cancer Institute, Inc., Boston, MA, USA). Antibodies used to stain murine MHC I, MHC II, CD80, and CD40 for flow cytometric analysis were purchased from eBioscience laboratories (San Diego, CA). RPMI 1640 medium, Dulbecco's modified Eagle medium, fetal bovine serum (FBS), penicillin/streptomycin, sodium pyruvate, and nonessential amino acids were obtained from Cellgro Mediatech (Herndon, VA).

\section{Preparation of Microparticles Using BSA}

The biodegradable nanoparticles were prepared following a method previously developed in our vaccine nanotechnology laboratory at Mercer University using the Buchi Mini Spray Dryer B-191 (22,25,27,29-34). Briefly, a 1\% solution of sterile BSA in sterile water was prepared and kept overnight for cross-linking with glutaraldehyde $(200 \mu \mathrm{L}$ for every $1 \mathrm{gm}$ of BSA). Excess glutaraldehyde was neutralized with sodium bisulfate the following day. Live attenuated measles virus 


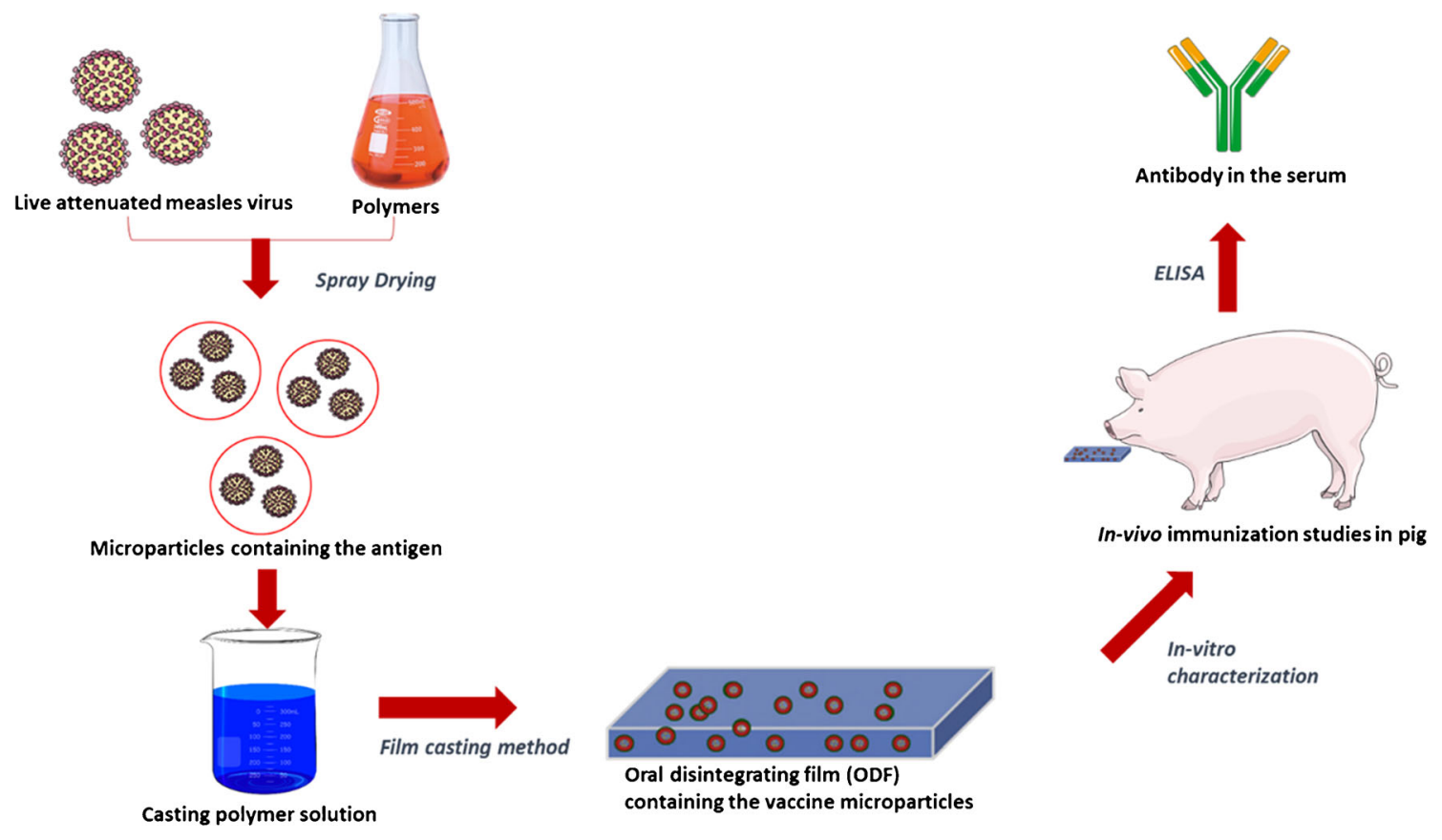

Fig. 1. Summary of the method of production of the orally disintegrating film (ODF) loaded with the vaccine microparticulate measles vaccine and further the immunization studies

(Edmonston Zagreb Strain) solution $(5 \%, w / w)$ was added to the solution and spray dried through a $0.5-\mathrm{mm}$ nozzle (nozzle temperature $-5^{\circ} \mathrm{C}$ ) at a flow rate of $20 \mathrm{~mL} / \mathrm{h}$ to obtain the measles vaccine loaded microparticles (30).
Microparticle Recovery Yield. Recovery yield of the microparticles after spray drying was calculated for all the batches formulated. Percent recovery yield was evaluated using the following formula:

Percentage Recovery Yield $(\%)=\frac{\text { Weight of microparticles after spray drying } * 100}{\text { Weight of all ingredients before spray drying }}$

Particle Size Distribution. Particle size of the optimized formulation was evaluated using the Spectrex Laser Particle Counter that works on the principle of laser diffraction. Two milligrams of the particles were suspended in 1-mL deionized water, vortexed well, and then analyzed by laser diffraction on the particle counter. Particle size was measured in triplicate for empty as well as antigen-loaded particles and contrasted.

Zeta Potential Measurement. Five micrograms of microparticles were suspended in $1 \mathrm{~mL}$ of deionized water, transferred to a zeta potential measurement cuvette, and measured using a Malvern Zetasizer. Zeta potential was measured in triplicate for the control formulation and contrasted with the antigen loaded microparticles.

Scanning Electron Microscopy of the Microparticles. Scanning electron microscopy (SEM) was performed to evaluate microparticle size distribution and surface morphology. Microparticles were mounted onto metal stubs using double-sided adhesive tape. After being vacuum-coated with a thin layer (100-150 $\AA$ ) of gold, the microparticles were examined by a scanning electron microscope Phenome benchtop SEM, Nanoscience Instruments, Phoenix, AZ.
Cytotoxicity Study. The toxicity of the measles vaccine microparticles toward murine RAW264 macrophages was examined in three replicates by the Alamar Blue assay $(25,30)$. Briefly, $2.5 \times 10^{5}$ cells were plated in each well of a 96-well plate, and vaccine microparticles ranging in concentration from 50 to $500 \mu \mathrm{g}, n=3$ for each concentration, were added to each well. Atropine sulfate was used as a positive control at a concentration of $10 \mathrm{mg} / \mathrm{mL}$. The readings were normalized with the blank microparticles. After $24 \mathrm{~h}, 10 \mu \mathrm{L}$ of a $10 \times$ solution of Alamar Blue was added to each well and plates were incubated for $4 \mathrm{~h}$ at $37^{\circ} \mathrm{C}$ following which the fluorescence was using BioTek Synergy H1 plate reader, Winooski, VT.

\section{Quantification of Antigen Presentation Molecules (MHC I and MHC II) Expression}

Dendritic cells (DC2.4) were plated at $3 \times 10^{5}$ cells per well in a 96 -well plate and incubated at $37^{\circ} \mathrm{C}$ for $24 \mathrm{~h}$ to adhere and stabilize. The adherent cells were pulsed with 250 $\mu \mathrm{g}$ measles vaccine microparticles containing $25 \mu \mathrm{g}$ of antigen in each well and incubated at $37^{\circ} \mathrm{C}$ for $16 \mathrm{~h}$. Equal amounts of 
blank microparticles without any antigen were used as controls in every experiment. The pulsed DC2.4 cells were then washed to remove extracellular microparticles prior to staining with fluorescein isothiocyanate (FITC) and phycoerythrin (PE) labeled MHC I and MHC II markers respectively (eBioscience laboratories, San Diego, CA) for $1 \mathrm{~h}$ at $4^{\circ} \mathrm{C}$. The stained DC2.4 cells were washed with Hank's Balanced Salt Solution (HBSS), and samples measurements were then quantified on BD Accuri C6 flow cytometer (BD Bioscience, San Jose, CA).

\section{Quantification of Co-stimulatory Molecules (CD40 and CD80)}

A similar method was used for the quantification of costimulatory molecules CD40 and CD80, DC2.4 were plated at $3 \times 10^{5}$ cells per well in a 96 -well plate and incubated at $37^{\circ} \mathrm{C}$ for $24 \mathrm{~h}$ to adhere and stabilize. The adherent cells were pulsed with $250-\mu \mathrm{g}$ measles vaccine microparticles containing $25 \mu \mathrm{g}$ of antigen in each well and incubated at $37^{\circ} \mathrm{C}$ for $16 \mathrm{~h}$. Equal amount of blank microparticles without any antigen was used as controls in every experiment. The pulsed DC2.4 cells were then washed to remove extracellular microparticles prior to staining with FITC labeled CD80 and APC labeled CD40 (eBioscience laboratories, San Diego, CA) for $1 \mathrm{~h}$ at $4^{\circ} \mathrm{C}$. The cells were then washed and samples measurements were acquired on BD Accuri C6 flow cytometer (BD Bioscience, San Jose, CA).

\section{Nitric Oxide Release from DC2.4 Cells}

Freshly grown adherent DC2.4 cells were harvested, washed, and re-suspended in Dulbecco's complete media, counted and adjusted to $10^{6} \mathrm{cell} / \mathrm{mL}$. Two hundred-microliter aliquots were then dispensed into each well of a 96-well plate at final $250 \times 10^{3}$ cell density prior to stimulation with measles vaccine microparticles and blank microparticles which served as blank control. The induced cells were incubated overnight at $37^{\circ} \mathrm{C}$ with $5 \% \mathrm{CO}_{2}$ and supernatants were harvested. Nitric oxide release was quantified using the Greiss chemical method as previously described (29). Briefly, the Griess chemical method was used to detect nitrite $\left(\mathrm{NO}_{2}\right)$ accumulated in supernatants of induced DC 2.4 cells. Griess reagent was freshly prepared by mixing equal volumes of $1 \%$ sulfanilamide and $0.1 \% \mathrm{~N}$-(1-naphthylethylenediamine) solutions. One hundred microliters of cell supernatants were transferred into a 96-well plate to which $100 \mu \mathrm{L}$ of Griess reagent was added. The plate was mixed gently, incubated for $10 \mathrm{~min}$ at room temperature and read at $540 \mathrm{~nm}$ using the EL312e microplate reader (BIO-TEK Instruments, Winooski, VT). The optical densities were correlated to the concentration of nitrite. Nitrite was quantitated using the standard curve of sodium nitrite (1-mM stock concentration in distilled water further diluted to the highest standard at $100 \mu \mathrm{M}$ followed by serial dilutions to $1.56 \mu \mathrm{M})(35)$.

\section{Preparation of the ODF}

The orally disintegrating films were cast based on the composition presented in Table I. Briefly, the film forming polymer Lycoat RS720 was dispersed in a solution containing precalculated amounts of water, Neosorb P60W, and Tween 80 under mechanical stirring. Measles vaccine microparticles previously prepared were uniformly dispersed into the previous suspension. This sample was then allowed to stand still until air bubbles formed during preparation dissipated. Thereafter, the ODF formulation loaded with vaccine microparticles was casted on a plastic support (byco-charts from BYC Gardner GmbH, Germany) using an automatic Erichsen Control Coater KCC101 equipped with a 200- $\mu \mathrm{m}$ wire wound rod applicator. The film was dried in a humidity chamber and when dry was cut into $3-\times 4-\mathrm{cm}$ film strips (15).

\section{Physicochemical Characterization of the ODF}

Film thickness was measured using a Mitutoyo screwtype micrometer with an accuracy of $\pm 3 \mu \mathrm{m}$ (Kanagawa, Japan). Tensile strength, Young's modulus, and percent elongation were determined using Stable Micro Systems' TA.XTplus Texture Analyzer (Surrey, England, U.K.) and the equations below:

$$
\begin{gathered}
\text { Tensile Strength }(\mathrm{MPa})=\frac{\text { Force at Breaking }(\mathrm{N})}{\text { Film Cross Sectional Area }\left(\mathrm{mm}^{2}\right)} \\
\text { Young's Modulus }(\mathrm{MPa})=\frac{\text { Force at Breaking }(\mathrm{N}) * \text { Initial Film Length }(\mathrm{mm})}{\text { Film Cross Sectional Area }\left(\mathrm{mm}^{2}\right) * \text { Change in Film Length }(\mathrm{mm})} \\
\text { Elongation } \%=\frac{\text { Change in Film Length }(\mathrm{mm})}{\text { Initial Film Length }(\mathrm{mm})} * 100
\end{gathered}
$$

Table I. Composition of the Measles Vaccine Microparticulate Loaded Orally Disintegrating Film (ODF)

\begin{tabular}{ll}
\hline Formulation & Ratio $(w / w)$ \\
\hline Measles vaccine microparticles & 0.2 \\
LYCOAT® RS 720 & 23 \\
Neosorb P60W & 5 \\
Tween 80 & 0.2 \\
\hline
\end{tabular}

\section{Porcine Methods}

The porcine studies were conducted in two domesticated, crossbred swine in the Purdue Translational Pharmacology (PTP) CTSI Core Facility under an approved Purdue University Animal Care and Use protocol (1112000407) (36). The PTP facility is registered with the USDA and OLAW and is housed in the School of Veterinary Medicine at 
Purdue. The domesticated pigs, approximately 5 months in age $(20 \mathrm{~kg}$; which have previously shown reveal PK properties similar to pediatric populations $(36,37)$, arrived into the Animal Housing Facility at Purdue and were acclimated for 7 days prior to any procedure. Pigs were housed for 8 weeks with access to food and water on a 12-h light cycle. Animals were fasted overnight prior to dosing. Animals were sedated with a combination of telazol, ketamine, and xylazine (TKX $50 \mathrm{mg} / \mathrm{mL}$ ) and maintained on $1-3 \%$ isoflurane via nose cone. Predose blood samples (1 $\mathrm{mL})$ were taken via jugular stick and placed in a vial containing EDTA. Each of the animals received 25 ODF films in the buccal pouch. The pouches were applied in sets of 2 , and once they dissolve, the next set of pouches was administered. Once all 25 films were inserted and dissolved, animals were removed from anesthesia and allowed to recover in a dark room. The ODFs were dosed at day 0 and 2 weeks later to provide a boost to the immune system. Animals were fed upon standing. Upon initiation of the study, the blood samples $(1 \mathrm{~mL})$ were collected into a vial containing EDTA at 2, 4, and 6 weeks after initial dosing for immunological evaluation and contrasted with naive pigs. Upon collection, each blood sample was stored at $-4^{\circ} \mathrm{C}$ for approximately $30 \mathrm{~min}$ until they were processed to separate plasma via centrifugation at $3400 \mathrm{rpm}$ for $10 \mathrm{~min}$. Plasma was siphoned off and placed in an Eppendorf tube for storage at $-80^{\circ} \mathrm{C}$ until shipping.

\section{Quantification of Serum Antibody Using ELISA}

The blood samples were collected prior to each dose of vaccination. Serum was isolated and analyzed for specific IgG titers for measles virus enzyme-linked immunosorbent assay (ELISA) (38). ELISA was performed by overnight coating of the live attenuated measles virus (vaccine antigen) on poly-Llysine coated high binding 96-well plate $(100 \mu \mathrm{g} /$ well in coating buffer) at $4{ }^{\circ} \mathrm{C}$. The plate was washed with washing solution (Tris $50 \mathrm{mM}, \mathrm{NaCl} 0.14 \mathrm{M}$, Tween $200.05 \%$ ) and blocked with $4 \%$ nonfat dry milk (Biorad, Hercules, CA) for $2 \mathrm{~h}$ at $37^{\circ} \mathrm{C}$. After washing, the plate was then incubated with 1:100 dilution of serum from vaccinated pigs. After $2 \mathrm{~h}$ of incubation followed by washing, HRP-tagged secondary antipig goat IgG (AbD Serotec $\AA$, Raleigh, NC) was added to each well, incubated for $1 \mathrm{~h}$ and then washed. TMB substrate reagent (3,3',5,5' -tetramethyl benzidine) (BD OptEIA ${ }^{\mathrm{TM}}$, BD Biosciences, CA) was added and the plate was again incubated for $30 \mathrm{~min}$ at $37^{\circ} \mathrm{C}$. The reaction was stopped by addition of $4 \mathrm{~N} \mathrm{H}_{2} \mathrm{SO}_{4}$. The plate was read and the absorbance values quantified at $450 \mathrm{~nm}$ using BioTek Synergy H1 microplate reader (BioTek instruments Inc., Winooski, VT).

\section{Statistical Analysis}

All experiments were performed in quadruplets, unless otherwise noted. Mean values $\pm \mathrm{SD}$ and $P$ value (Student's $t$ test unpaired, two-tail distribution) were determined individually for all experiments with Microsoft Excel software. A $P$ value of less than 0.05 was considered to be statistically significant.

\section{RESULTS}

\section{Formulation and Characterization of Measles Microparticulate Vaccine}

Microparticles Formulation and Recovery Yield. The yield of spray drying process was within a range of $85-95 \%$ $(w / w)$ (Table II). The loss during microparticle preparation can be attributed to microparticles sticking to the cylinder and cyclone of the spray dryer.

Particle Size Analysis. The particle size distribution of novel vaccine microparticle formulations from two different batches of empty particles and measles antigen loaded microparticles was investigated using Spectrex laser counter (Spectrex Corporation). There was no significant difference in size between empty and measles vaccine loaded microparticles $\sim 90 \%$ of which were between 1 and $5 \mu \mathrm{m}$ with an average particle size of $3.65 \pm 1.89 \mu \mathrm{m}$ (Table II).

Zeta Potential Measurements. Zeta potential is indicative of the surface charge of the particle. A high positive or negative charge indicates good stability and suspendability of the particle when reconstituted in media as it avoids agglomeration (30). The zeta potential measurements of empty (unloaded) and antigen-loaded microparticle suspensions in deionized water were in the range of -30 to $-35 \mathrm{mV}$ with the mean of $-32.65 \pm 2.4 \mathrm{mV}$ and did not differ significantly from each other (Table II).

Scanning Electron Microscopy. The surface morphology of the formulated microparticles was investigated using scanning electron microscopy. The surface of the microparticles was irregularly shaped and rough (Fig. 2). The different shapes of the microparticles may be helpful for biodistribution and uptake by macrophages (39-41).

Cytotoxicity Study. To assess the cytotoxicity of the formulated vaccine-loaded microparticles on macrophages, we employed the Alamar Blue assay $(25,30)$. The results of the cytotoxicity study indicated that the formulation was not toxic to murine macrophages RAW264 at doses ranging from 50 to $500 \mu \mathrm{g}$ per well (Fig. 3). The viability of the cell population exposed to different doses of microparticles did not differ significantly from the cell populations not exposed to microparticles indicating that the microparticles were not toxic to the cells (Fig. 3). Atropine sulfate was used as a positive control and as expected showed highly decreased

Table II. Physical Characteristics of the Measles Vaccine Microparticles

\begin{tabular}{lll}
\hline & Range & Mean $\pm \mathrm{SD}$ \\
\hline Recovery yield $(\%)$ & 10 & $92.76 \pm 4.5$ \\
Particle Size $(\mu \mathrm{m})$ & 4 & $3.65 \pm 1.89$ \\
Zeta Potential $(\mathrm{mV})$ & 5 & $-32.65 \pm 2.4$ \\
\hline
\end{tabular}

The recovery yield (\%) after the spray drying process, particle size, and the zeta potential was measured in triplicates, and mean and the standard deviation are reported along with the range 


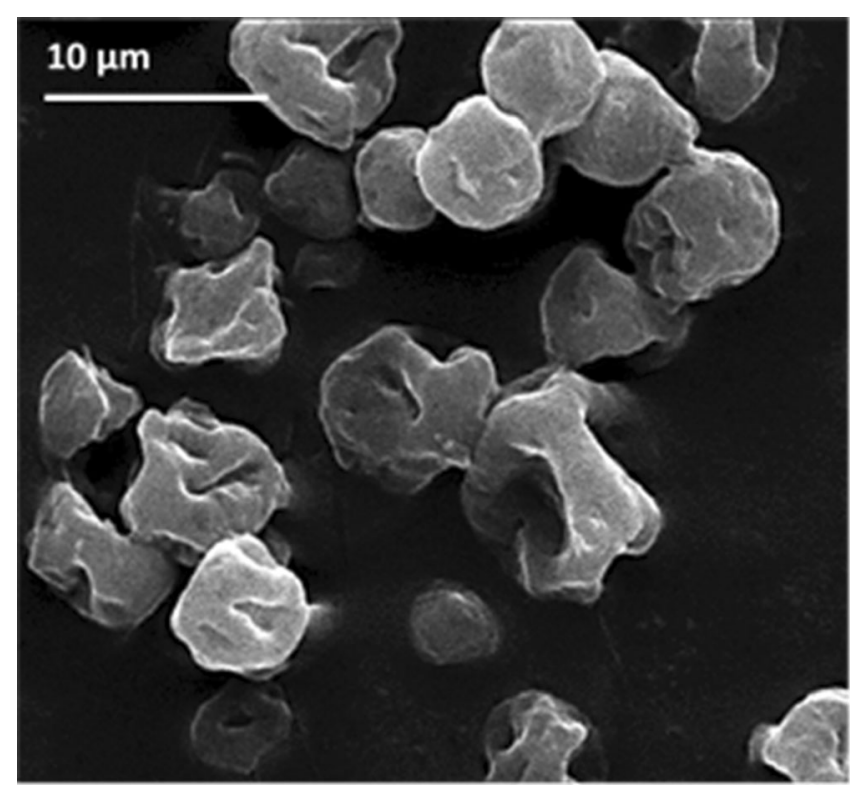

Fig. 2. Scanning electron microscope (SEM) image of the measles vaccine microparticles

viability in comparison to the negative control, i.e., cells alone. The results indicate that measles vaccine-loaded microparticles are not toxic to macrophages.

Antigen Presentation on Dendritic Cells. The primary requirement for a vaccine to show its effect by activating the dendritic cell after the antigen processing and expressing the antigen on the major histocompatibility class I and II molecules. Antigen presentation by matured dendritic cells to $\mathrm{T}$ lymphocytes is essential for vaccine-induced protective immune responses. Thus, we assessed the expression of the MHC class I and MHC class II molecules on the surface of dendritic cells when exposed to the vaccine microparticles. Our results show that there is a significant increase in the expression of both MHC I and MHC II molecules when exposed to vaccine microparticles as compared to blank microparticles (Fig. 4).

Co-stimulatory Molecules Expression on Dendritic Cells. Co-stimulatory molecules provide the crucial second signal to naïve $\mathrm{T}$ cells for its activation and proliferation leading to subset differentiation and effector functions required to elicit adaptive immune responses $(29,42,43)$. Since effective antigen presentation by mature dendritic cells to $\mathrm{T}$ cells requires the expression of co-stimulatory molecules such as CD40 and CD80, we assessed their expression in dendritic cells pulsed with measles vaccine microparticles and blank microparticles. We found that measles vaccine microparticles enhanced the expression of co-stimulatory molecules CD40 and CD80 on dendritic cells when compared to blank microparticles (Fig. 5).

Nitric Oxide Release from the Dendritic Cells. Nitric oxide (NO) is an innate immune marker which is released after the uptake and processing of the vaccine antigens by the dendritic cells. Nitric oxide is also an important innate immunity marker reflecting the antigen recognition and stimulation of dendritic cells. A higher level of NO release indicates a stronger activation of dendritic cells. We observed a significant higher level of NO released by the dendritic cells when compared to the blank microparticles (Fig. 6). This also suggests that BSA cross-linked polymer matrix used to make the microparticles is not immunogenic and the innate immune response generated can be attributed to the measles antigen present the vaccine loaded microparticle group.

Vaccine Loaded ODF Characterization. Pharmaceutical films must endure different types of mechanical stress encountered during manufacture, packaging, travel, and consumer handling. The physical characteristics of the vaccine loaded ODF are listed in Table III. Briefly, undamaged films with an average width of $30 \mathrm{~mm}$ and thickness of $79 \mu \mathrm{m}$ were

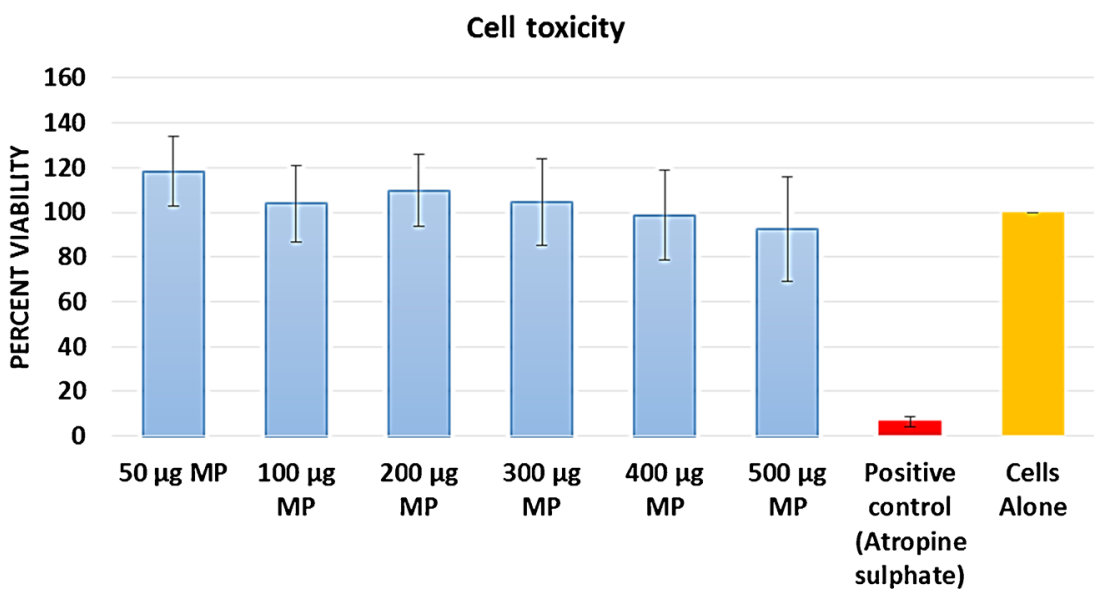

Fig. 3. Albumin-based microparticles are not toxic to macrophages. Briefly, RAW264 murine macrophages treated with increasing doses of microparticles and incubated overnight. Microparticles by themselves were not toxic to RAW264 cells as compared to untreated cells. The cytotoxicity was analyzed by the Alamar Blue assay that uses the reducing power of living cells to quantitatively measure cell viability. Experiment was performed in triplicate 


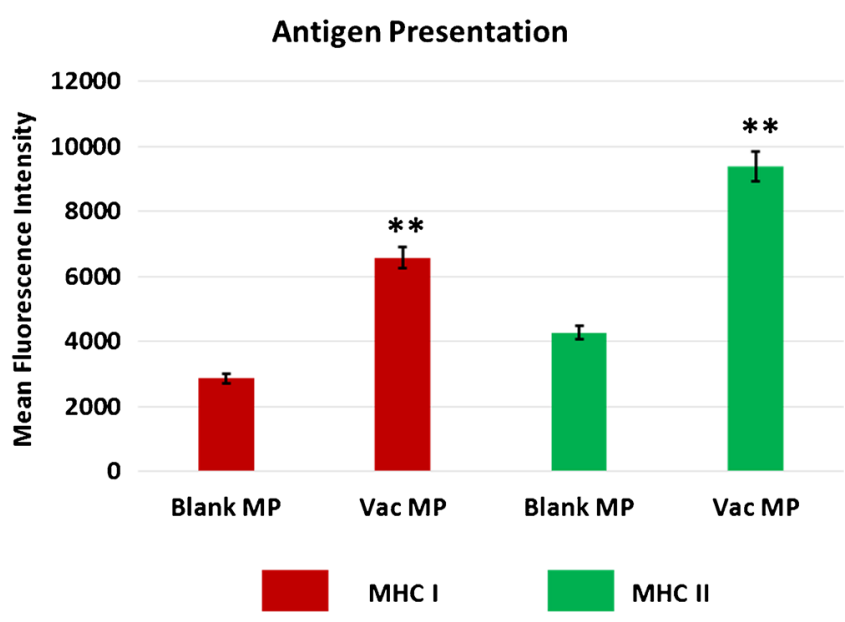

Fig. 4. Antigen-induced expression of MHC I and MHC II complex on murine dendritic cells is presented. Briefly, murine dendritic cells $\left(3 \times 10^{5}\right)$ were pulsed with measles vaccine microparticles (antigen dose $25 \mu \mathrm{g} / 3 \times 10^{5}$ cells) for $16 \mathrm{~h}$. The expression of antigen presenting receptor MHC I (red bars) and MHC II (green bars) was detected using flow cytometer following staining with FITC MHC I and FITC MHC II markers. Error bars represent the standard deviation from the mean of four independent experiments. $P$ values were calculated using Student's $t$ test unpaired, two-tail distribution comparing to the blank MP and microparticulate vaccine. $* * P<0.01$ was significant

placed between two rubber grippers approximately $21 \mathrm{~mm}$ apart. Stress was applied at $10 \mathrm{~mm} / \mathrm{min}$ until rupture. Studies were conducted with four replicates. Mechanical properties of the vaccine loaded ODF are listed in Table IV. The transverse section of the vaccine loaded ODF is shown in Fig. 7 where the white spots are the vaccine microparticles which are uniformly dispersed throughout the matrix of the film.

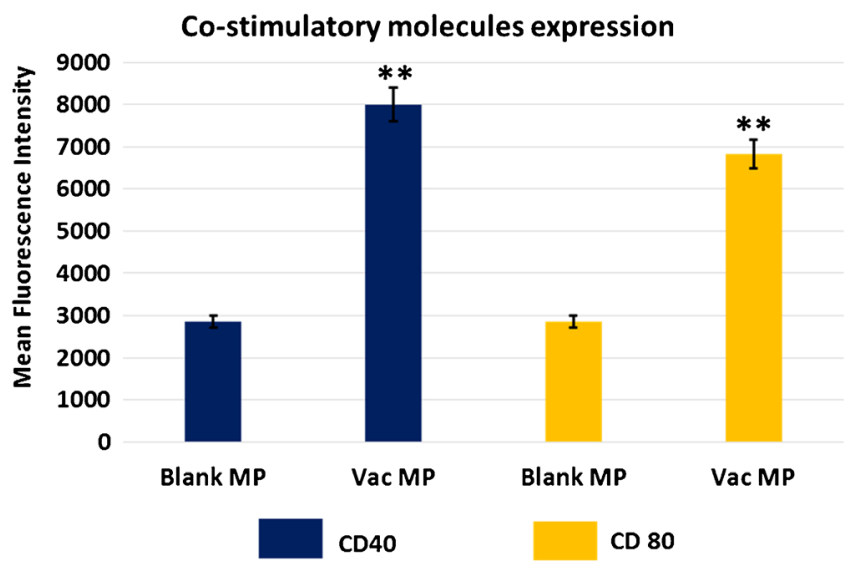

Fig. 5. The results from the expression of co-stimulatory molecules, CD 40 and CD 80, on dendritic cells are illustrated. Murine dendritic DC 2.6 cells $\left(3 \times 10^{5}\right)$ pulsed with measles vaccine microparticles (antigen dose $25 \mu \mathrm{g} / 3 \times 10^{5}$ cells) for $16 \mathrm{~h}$. The expression of costimulatory molecules CD40 (blue bars) and CD80 (yellow bars) on dendritic cells was detected using flow cytometer following staining with APC CD40 markers and FITC CD80. Error bars represent the standard deviation from the mean of four independent experiments. $P$ values were calculated using Student's $t$ test unpaired, two-tail distribution comparing to the blank MP and microparticulate vaccine. $* * P<0.01$ was significant

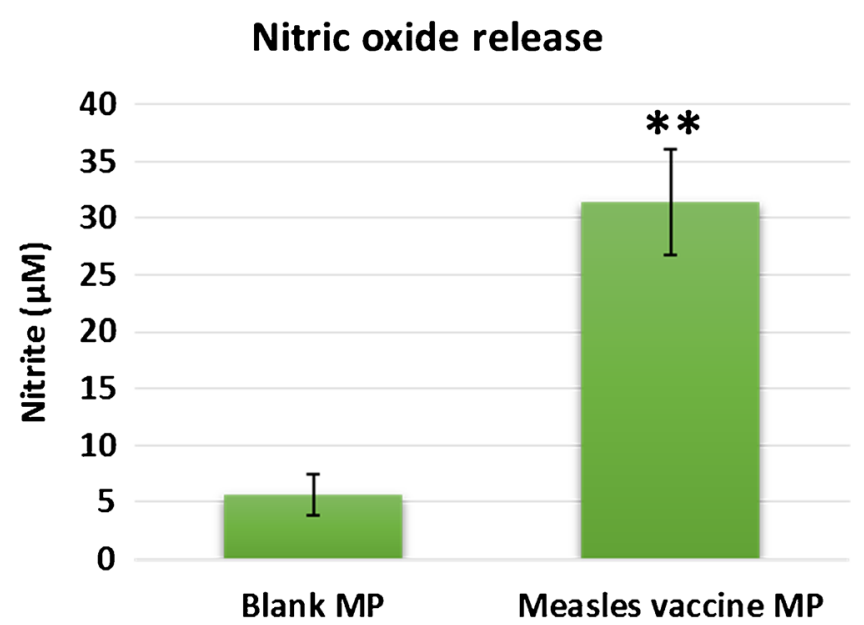

Fig. 6. Nitric oxide release from dendritic cells was measured. Briefly, murine dendritic cells (DC 2.4) $\left(250 \times 10^{3}\right)$ were pulsed with measles vaccine microparticles (antigen dose $25 \mu \mathrm{g} / 250 \times 10^{3}$ cells) for $16 \mathrm{~h}$. Nitrite accumulation in the supernatants was determined using the Greiss reagent. There is significant release of nitric oxide release from the measles vaccine microparticles when compared to blank microparticles. Error bars represent the standard deviation from the average of two independent determinations per well. The data shown are representative of three independent experiments. $* * P$ value $<0.05$ was calculated as above

Immunization Studies. To assess the ability of the measles vaccine loaded on to the ODF, the ODF was given to pigs via the buccal route. We chose the juvenile porcine model to test our vaccine formulation because the oral mucosa of pigs resembles that of human more closely than any other animal in terms of structure and composition $(44,45)$. Moreover, pigs also have nonkeratinized buccal mucosa similar to that of human $(46,47)$. The presence of measles specific antibody in the serum samples was assessed using ELISA as mentioned. The increase in the quantity of measles specific antibody was compared to the serum collected prior to dosing (naïve pigs). There was a significant increase in antibody titers after 2 weeks of the first dose. After the second dose at 2 weeks, a further increase in the antibody titer was observed in weeks 4 and 6 . The antibody levels remained significantly higher at all the time points (weeks 2, 4, and 6) when contrasted with predose levels (Fig. 8).

\section{DISCUSSION}

There is an imperative need for the development of novel vaccine delivery strategies in order to improve the

Table III. Physical Characterization of the Measles ODF Vaccine

\begin{tabular}{ll}
\hline Parameter & Value \\
\hline Length & $2.0 \mathrm{~cm}$ \\
Width & $3.0 \mathrm{~cm}$ \\
Thickness & $0.079 \mathrm{~mm}$ \\
\hline
\end{tabular}


Table IV. Mechanical Properties of the Films Are Listed as the Average and the Standard Deviation

\begin{tabular}{ll}
\hline Parameter & Value (SD) \\
\hline Tensile strength (MPa) & $15.8(2.4)$ \\
Young's modulus (MPa) & $739(192)$ \\
Elongation (\%) & $2.2(0.4)$ \\
\hline
\end{tabular}

patient compliance and effective immunization on large-scale populations. Orally disintegrating films (ODF) containing measles vaccine microparticles are one such method of a noninvasive immunization strategy. Being by design a fast melting formulation (few seconds), the ODF dissolves at the first contact with saliva and facilitates the coating of the buccal surface enhancing the delivery of the vaccine into the buccal mucosae. ODF also assures dosing accuracy and elicits the induction of an effective immune response. As the buccal mucosae are rich in immune cells such as dendritic cells and Langerhans cells, it is a very convenient and patient compliant site for vaccination. This is supported by data including that of Lundholm et al. demonstrating that pDNA administered to the oral cheek of mice using a jet injection induced the production of IgA signifying a mucosal immune response (48). In addition, Wang et al. reported that mucosal delivery of a melanoma vaccine in a hamster model helped treat oral melanoma and distant skin lesions (49). All of these studies demonstrated that buccal immunization is possible and can be very effective at the same time.

Despite tremendous achievements in global measles control, the virus still circulates, causing infection, severe disease, and death. Since measles virus is highly contagious, high levels of population immunity are required in order to interrupt measles virus transmission (3). This poses a challenge on health care practitioners to immunize large populations, as the current vaccine is invasive and requires medical professionals for its administration $(5,6,50)$. This problem could be overcome using an ODF measles vaccine as it can be administered by the individual themselves. Thus, the cost associated with administration and cold chain storage
Serum Antibody levels

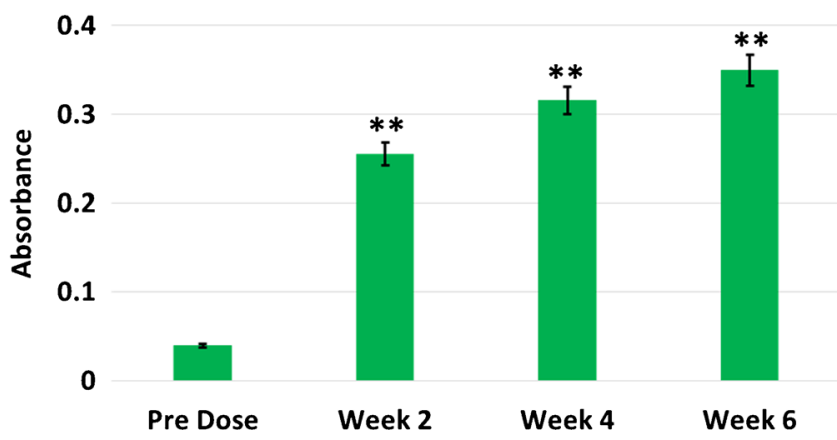

Fig. 8. $\operatorname{IgG}$ levels post-immunization were assessed in the juvenile porcine model and contrasted with predose levels in naïve pigs. Briefly, blood samples were collected before each dose and serum was separated. The serum antibody levels were measured using ELISA. There is a significant increase in the serum IgG levels when compared between serum IgG level predosing and post-dosing $(* * P<0.01)$. Error bars represent the standard deviation from the average of two independent determinations per well. The data shown are representative of three independent experiments $(* * P<0.01)$

could be minimized, and large population could be immunized at an affordable cost.

Microparticles offer a novel delivery system for the vaccines and have more recently been utilized for both infectious disease and cancer vaccines (22,24,25,27,30,32,51). The microparticles are made from a biocompatible and biodegradable polymer matrix which protects the antigen (measles virus) in a stable form. The microparticulate matrix is not toxic to normal cells at higher concentrations and does not induce an immune response. These microparticles due to their size are easily taken up by the antigen presenting cells and thus help in better antigen presentation to the immune system (29). Any adjuvants, such as alum and MF59, can also be encapsulated in these microparticles to improve the efficacy of the vaccine and generate a robust immune response (30).

In our study, we found that the measles vaccine when formulated as microparticles and incorporated into the ODF
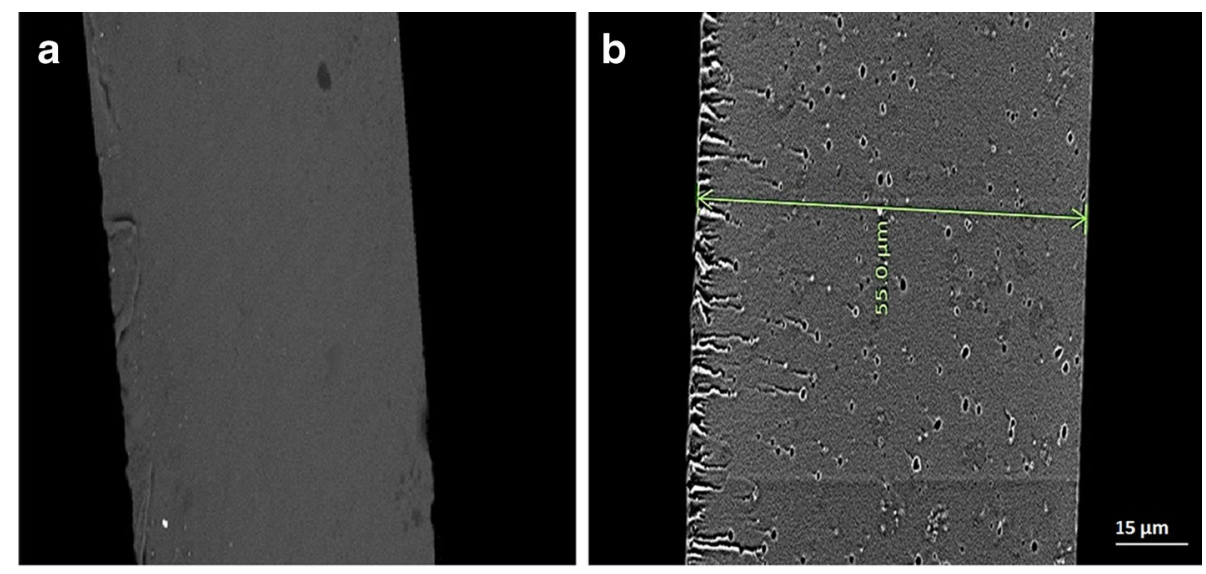

Fig. 7. Transverse section scanning electron microscope (SEM) image of the orally disintegrating film (ODF) is illustrated. a Blank ODF, i.e., without the microparticles. b ODF loaded with measles vaccine microparticles. The ODFs are of uniform thickness with the microparticulate vaccine embedded (white and black holes) in the film matrix 
formulation as an oral delivery system can induce significantly higher antigen presentation to the MHC I and II molecules and also the corresponding co-stimulatory molecules CD40 and CD80 when compared to blank formulation. This ensures that the ODF, after dissolving in the oral cavity, is coating the buccal mucosa allowing the vaccine microparticles to be taken up by the APCs and stimulate both the arms of the immune system via the Th1 and Th2 pathways. Due to this activation, we found significant high antibody titer in the juvenile porcine immunization model study (36). Thus, from this exploratory study to determine if the juvenile porcine buccal mucosa can serve as a surrogate for human response and an alternative to invasive vaccines using the ODF formulations, we plan to test other vaccines against influenza, meningitis, etc. in similar formulations in the future. We also plan to further develop the current measles vaccine ODF to increase loading and optimize efficacy in a readily translatable formulation.

\section{CONCLUSION}

The novel in vivo approach to formulate microparticulate measles vaccine in an orally disintegrating film for delivery via the buccal route in a juvenile porcine model has revealed a promising mode of immunization strategy against measles in a commonly accepted surrogate model for human buccal delivery (36). As mentioned earlier, this mode of immunization can be used for large-scale immunizations to prevent transmission of measles virus and epidemics. Moreover, it is patient compliant due to its ease of administration and can be given to populations of all ages from infants to adults. In this exploratory study, we conducted immunization studies with the ODF measles vaccine in juvenile porcine model and found significant increase in the antibody titers along with induction of innate immune response.

\section{ACKNOWLEDGMENTS}

The authors would like to acknowledge that all of porcine studies were conducted in the Purdue Translational Pharmacology (PTP) Core Facility with funding from the Clinical Translational Sciences Award (Core Pilot Funding NIH Grant no. RR025761).

\section{REFERENCES}

1. Moss WJ, Griffin DE. Measles. Lancet Lond Engl. 2012;379(9811):153-64.

2. Clements CJ, Cutts FT. The epidemiology of measles: thirty years of vaccination. Curr Top Microbiol Immunol. 1995;191:1333 .

3. Goodson JL, Seward JF. Measles 50 years after use of measles vaccine. Infect Dis Clin N Am. 2015;29(4):725-43.

4. What you should know about the latest measles outbreaks [internet]. About.com Health. [cited 2016 Jan 12]. Available from: http://pediatrics.about.com/od/measles/a/measlesoutbreaks.htm.

5. Stalkup JR. A review of measles virus. Dermatol Clin. 2002;20(2):209-215, v.

6. Griffin DE, Pan C-H, Moss WJ. Measles vaccines. Front Biosci J Virtual Libr. 2008;13:1352-70.
7. Griffin DE, Ward BJ, Esolen LM. Pathogenesis of measles virus infection: an hypothesis for altered immune responses. J Infect Dis. 1994;170 Suppl 1:S24-31.

8. Griffin DE, Lin W-H, Pan C-H. Measles virus, immune control, and persistence. FEMS Microbiol Rev. 2012;36(3):649-62.

9. Garly M-L, Aaby P. The challenge of improving the efficacy of measles vaccine. Acta Trop. 2003;85(1):1-17.

10. Griffin DE, Pan CH. Measles: old vaccines, new vaccines. Curr Top Microbiol Immunol. 2009;330:191-212.

11. Godlee F, Smith J, Marcovitch H. Wakefield's article linking MMR vaccine and autism was fraudulent. BMJ. 2011;342:c7452.

12. Kalkbrenner AE, Schmidt RJ, Penlesky AC. Environmental chemical exposures and autism spectrum disorders: a review of the epidemiological evidence. Curr Probl Pediatr Adolesc Health Care. 2014;44(10):277-318.

13. Madsen KM, Hviid A, Vestergaard M, Schendel D, Wohlfahrt J, Thorsen $\mathrm{P}$, et al. A population-based study of measles, mumps, and rubella vaccination and autism. $N$ Engl $J$ Med. 2002;347(19):1477-82.

14. Taylor LE, Swerdfeger AL, Eslick GD. Vaccines are not associated with autism: an evidence-based meta-analysis of case-control and cohort studies. Vaccine. 2014;32(29):3623-9.

15. Lou H, Liu M, Qu W, Hu Z, Brunson E, Johnson J, et al. Evaluation of chlorpheniramine maleate microparticles in orally disintegrating film and orally disintegrating tablet for pediatrics. Drug Dev Ind Pharm. 2014;40(7):910-8.

16. Cui Z, Mumper RJ. Bilayer films for mucosal (genetic) immunization via the buccal route in rabbits. Pharm Res. 2002;19(7):947-53.

17. Amorij J-P, Kersten GFA, Saluja V, Tonnis WF, Hinrichs WLJ, Slütter B, et al. Towards tailored vaccine delivery: needs, challenges and perspectives. J Control Release. 2012;161(2):363-76.

18. Kraan H, Vrieling H, Czerkinsky C, Jiskoot W, Kersten G, Amorij J-P. Buccal and sublingual vaccine delivery. J Control Release. 2014;190:580-92.

19. Liew KB, Tan YTF, Peh KK. Characterization of oral disintegrating film containing donepezil for Alzheimer disease. AAPS PharmSciTech. 2012;13(1):134-42.

20. Kweon M-N. Sublingual mucosa: a new vaccination route for systemic and mucosal immunity. Cytokine. 2011;54(1):1-5.

21. Alayoubi A, Haynes L, Patil H, Daihom B, Helms R, Almoazen H. Development of a fast dissolving film of epinephrine hydrochloride as a potential anaphylactic treatment for pediatrics. Pharm Dev Technol. 2016;7:1-5.

22. Tawde SA, Chablani L, Akalkotkar A, D'Souza C, ChirivaInternati M, Selvaraj P, et al. Formulation and evaluation of oral microparticulate ovarian cancer vaccines. Vaccine. 2012;30(38):5675-81.

23. Chablani L, Tawde SA, Akalkotkar A, D'Souza C, Selvaraj P, D'Souza MJ. Formulation and evaluation of a particulate oral breast cancer vaccine. J Pharm Sci. 2012;101(10):3661-71.

24. Bhowmik T, D'Souza B, Shashidharamurthy R, Oettinger C, Selvaraj P, D'Souza MJ. A novel microparticulate vaccine for melanoma cancer using transdermal delivery. J Microencapsul. 2011;28(4):294-300.

25. Shastri PN, Kim M-C, Quan F-S, D’Souza MJ, Kang S-M. Immunogenicity and protection of oral influenza vaccines formulated into microparticles. J Pharm Sci. 2012;101(10):362335.

26. Lai YH, D'Souza MJ. Formulation and evaluation of an oral melanoma vaccine. J Microencapsul. 2007;24(3):235-52.

27. Uddin AN, Bejugam NK, Gayakwad SG, Akther P, D’Souza MJ. Oral delivery of gastro-resistant microencapsulated typhoid vaccine. J Drug Target. 2009;17(7):553-60.

28. D'Souza B, Bhowmik T, Shashidharamurthy R, Oettinger C, Selvaraj P, D'Souza M. Oral microparticulate vaccine for melanoma using M-cell targeting. J Drug Target. 2012;20(2):166-73.

29. Ubale RV, Gala RP, Zughaier SM, D'Souza MJ. Induction of death receptor CD95 and co-stimulatory molecules CD80 and CD86 by meningococcal capsular polysaccharide-loaded vaccine nanoparticles. AAPS J. 2014;16(5):986-93.

30. Ubale RV, D’Souza MJ, Infield DT, McCarty NA, Zughaier SM. Formulation of meningococcal capsular polysaccharide vaccine- 
loaded microparticles with robust innate immune recognition. J Microencapsul. 2013;30(1):28-41.

31. Bejugam NK, Uddin AN, Gayakwad SG, D'Souza MJ. Formulation and evaluation of albumin microspheres and its enteric coating using a spray-dryer. J Microencapsul. 2008;25(8):577-83.

32. Singh M, Salnikova M. Novel approaches and strategies for biologics, vaccines and cancer therapies. Cambridge: Academic Press, Elsevier; 2015. 520 p.

33. Bejugam NK, Gayakwad SG, Uddin AN, D'Souza MJ. Microencapsulation of protein into biodegradable matrix: a smart solution cross-linking technique. J Microencapsul. 2013;30(3):274-82.

34. Chablani L, Tawde SA, D'Souza MJ. Spray-dried microparticles: a potential vehicle for oral delivery of vaccines. J Microencapsul. 2012;29(4):388-97.

35. Zughaier SM, Tzeng Y-L, Zimmer SM, Datta A, Carlson RW, Stephens DS. Neisseria meningitidis lipooligosaccharide structure-dependent activation of the macrophage CD14/Tolllike receptor 4 pathway. Infect Immun. 2004;72(1):371-80.

36. Roth WJ, Kissinger CB, McCain RR, Cooper BR, MarchantForde JN, Vreeman RC, et al. Assessment of juvenile pigs to serve as human pediatric surrogates for preclinical formulation pharmacokinetic testing. AAPS J. 2013;15(3):763-74.

37. Kulkarni R, Yumibe N, Wang Z, Zhang X, Tang CC, Ruterbories $\mathrm{K}$, et al. Comparative pharmacokinetics studies of immediateand modified-release formulations of glipizide in pigs and dogs. $\mathrm{J}$ Pharm Sci. 2012;101(11):4327-36.

38. Plans $\mathrm{P}$, de Ory F, Campins M, Álvarez E, Payà T, Guisasola E, et al. Prevalence of anti-rubella, anti-measles and anti-mumps IgG antibodies in neonates and pregnant women in Catalonia (Spain) in 2013: susceptibility to measles increased from 2003 to 2013. Eur J Clin Microbiol Infect Dis. 2015;34(6):1161-71.

39. Agarwal R, Singh V, Jurney P, Shi L, Sreenivasan SV, Roy K. Scalable imprinting of shape-specific polymeric nanocarriers using a release layer of switchable water solubility. ACS Nano. 2012;6(3):2524-31.

40. Agarwal R, Roy K. Intracellular delivery of polymeric nanocarriers: a matter of size, shape, charge, elasticity and surface composition. Ther Deliv. 2013;4(6):705-23.
41. Glangchai LC, Caldorera-Moore M, Shi L, Roy K. Nanoimprint lithography based fabrication of shape-specific, enzymaticallytriggered smart nanoparticles. J Control Release. 2008;125(3):263-72.

42. McAdam AJ, Schweitzer AN, Sharpe AH. The role of B7 costimulation in activation and differentiation of CD4+ and CD8+ T cells. Immunol Rev. 1998;165:231-47.

43. Fuse S, Tsai C-Y, Rommereim LM, Zhang W, Usherwood EJ. Differential requirements for CD80/86-CD28 costimulation in primary and memory CD4 T cell responses to vaccinia virus. Cell Immunol. 2011;266(2):130-4.

44. Squier CA, Cox PS, Wertz PW, Downing DT. The lipid composition of porcine epidermis and oral epithelium. Arch Oral Biol. 1986;31(11):741-7.

45. Squier CA, Cox P, Wertz PW. Lipid content and water permeability of skin and oral mucosa. J Investig Dermatol. 1991;96(1):123-6.

46. Junginger HE, Hoogstraate JA, Verhoef JC. Recent advances in buccal drug delivery and absorption-in vitro and in vivo studies. J Control Release. 1999;62(1-2):149-59.

47. Hoogstraate AJ, Verhoef JC, Tuk B, Pijpers A, van Leengoed $\mathrm{LA}$, Verheijden $\mathrm{JH}$, et al. In-vivo buccal delivery of fluorescein isothiocyanate-dextran 4400 with glycodeoxycholate as an absorption enhancer in pigs. J Pharm Sci. 1996;85(5):457-60.

48. Lundholm P, Asakura Y, Hinkula J, Lucht E, Wahren B. Induction of mucosal $\operatorname{IgA}$ by a novel jet delivery technique for HIV-1 DNA. Vaccine. 1999;17(15-16):2036-42.

49. Wang J, Murakami T, Hakamata Y, Ajiki T, Jinbu Y, Akasaka Y, et al. Gene gun-mediated oral mucosal transfer of interleukin 12 cDNA coupled with an irradiated melanoma vaccine in a hamster model: successful treatment of oral melanoma and distant skin lesion. Cancer Gene Ther. 2001;8(10):705-12.

50. Brniak W, Maślak E, Jachowicz R. Orodispersible films and tablets with prednisolone microparticles. Eur J Pharm Sci. 2015;75:81-90.

51. Nanoparticulate Vaccine Delivery Systems [Internet]. CRC Press. [cited 2015 Dec 16]. Available from: https:// www.crcpress.com/Nanoparticulate-Vaccine-Delivery-Systems/ DSouza/9789814613309 